\title{
BIBLIOGRAFÍA ODONTOLÓGICA COLOMBIANA
}

\author{
Jaime Alberto Castro N.
}

Odontólogo, U. de Antioquia.

Miembro de la Academia Cordobesa de Historia de la Odontología

Autor responsable de correspondencia: Dr. Jaime Alberto Castro N.

Correo electrónico: jacastron@hotmail.com

\begin{abstract}
RESUMEN
El propósito de este artículo es hacer un análisis de la cantidad de libros publicados en Colombia sobre odontología. Colombia es uno de los países más atrasados en América Latina en cuanto a investigación y producción científica se refiere. La profesión odontológica no ha sido ajena a esta situación. El primer libro publicado en Colombia sobre odontología fue Observaciones sobre la estructura, fisiolojía (sic), anatomía y enfermedades de los dientes escrito por el norteamericano Joseph Watson ver Valen, publicado en Bogotá, en 1849. Desde esa fecha, hasta el presente (2004), han transcurrido 155 años, durante los cuales se han publicado 100 títulos. La mayoría de las obras han sido publicadas en Bogotá y Medellín (92\%). De acuerdo con la discriminación por géneros, el 79\% de los libros han sido publicados por hombres. [Castro JA. Bibliografía odontológica colombiana. Ustasalud Odontología 2004; 3: 55 - 60]
\end{abstract}

Palabras clave: Odontología, Bibliografía.

\section{COLOMBIAN DENTAL LITERATURE}

\begin{abstract}
The purpose of this paper is to do an analysis concerning the amount of books published in Colombia on Dentistry. Colombia is one of the Latin American countries in which both research and scientific production still latecomer. Dental profession has not been free from this situation. The first book published in Colombia on Dentistry was Dental technique or observations on the structure, physiology, anatomy and dental diseases, written by American Joseph Watson ver Valen and released in Bogotá in 1849. In 155 years there have been released 100 books, the great majority of them in Bogota and Medellin (92\%). According to the gender, $79 \%$ of the books have been written by men.
\end{abstract}

Key words: Dentistry, Bibliography.

Recibido para publicación: 20 de febrero de 2004. Aceptado para publicación: 10 de mayo de 2004.

\section{INTRODUCCIÓN}

La producción de textos odontológicos en Colombia comenzó 39 años antes de la aparición del Colegio Dental de Bogotá, fundado en 1888. Fue la iniciativa del odontólogo norteamericano, radicado en Colombia, Joseph Watson ver Valen, que publicó en el país el primer libro sobre odontología. En efecto, en 1849 este profesional publicó en Bogotá la obra Observaciones sobre la estructura, fisiolojía (sic), anatomía y enfermedades de los dientes.

Posterior a su obra se publicaron muy escasos títulos sobre el tema odontológico, de modo que en el siglo XIX tan sólo vieron la luz pública cuatro títulos:

- Observaciones sobre la estructura, fisiolojia (sic), ana- tomía y enfermedades de los dientes. Joseph Watson ver Valen. Bogotá. 1849.

- Manual del dentista. José Peregrino Sanmiguel. Bogotá. 1866.

- Estudios dentales. Guillermo Vargas Paredes. Bogotá. 1892. - Lecciones de materia médica y terapéutica dentales. Alberto Restrepo. Bogotá. 1895.

En el siglo XX la producción mejoró, a mediados de la centuria y al final de la misma. A pesar de que la investigación científica y la producción de material escrito en Colombia ha mostrado un crecimiento vertiginoso desde la década del noventa, todavía el país se encuentra por debajo de otros en América Latina, como Brasil, México, Chile y Venezuela. 


\section{METODOLOGÍA}

Debido a que en Colombia no existe una base de datos ni escrita ni electrónica, que mantenga una información actualizada acerca de los libros que se publican sobre odontología, se procedió a realizar un inventario de las obras de acuerdo con tres grandes fuentes:

1. Libros. Se recurrió a los trabajos de Echeverri (1952), Payares y Arango (1991), Herazo Acuña (1994) y Duque y López (2002) sobre la historia de la odontología en Colombia.

2. Bibliotecas. Se hizo una búsqueda detallada en las bibliotecas de las siguientes universidades: Universidad del Sinú, Universidad de Cartagena, Pontificia Universidad Javeriana, Universidad de Antioquia e Instituto de Ciencias de la Salud (CES).

3. Librerías. En la Librería Nacional (Bogotá), Domus Libri (Montería) y Librería Científica (Medellín). Se buscaron en la sección de odontología todos los ejemplares sobre este campo.

Con estas tres grandes fuentes se procedió al inventario y posterior realización de la base de datos donde con las siguientes características:

- Nombre del autor.

- Título.

- Ciudad de publicación.

- Año de publicación.

Las publicaciones fueron divididas de acuerdo con temas específicos y organizadas en orden cronológico de publicación. Se analizaron tópicos como cantidad y género de los autores, año y ciudad de publicación.

\section{RESULTADOS}

En 155 años de literatura odontológica en Colombia, contados a partir de la publicación del libro de Joseph Watson ver Valen en 1849, tan sólo se han publicado 100 libros. Las especialidades sobre las cuales se han publicado más ejemplares son la epidemiología con 15 libros y la historia de la odontología con 10. Entre las especialidades menos trabajadas están la cirugía maxilofacial, periodoncia, odontología forense, farmacología y radiología. A continuación ofrecemos una lista detallada de los ejemplares encontrados (Tabla 1).

\section{Colecciones}

1. Guías de práctica clínica basadas en la evidencia. ISSACFO. Manizales, 1998.

\section{Odontología forense}

1. Odontología forense. José Vicente Rodríguez, Héctor Polanco, Yesid Valdés y Alfonso Casas. Bogotá, 1995.

2. Odontoestomatología forense. Antonio Guerra. Bogotá, 2002.

\section{Radiología}

1. Interpretación radiológica dental. Álvaro Delgado Morales. Bogotá, 1956.

2. Radiodoncia. Álvaro Delgado Morales y Álvaro Delgado Hernández. Bogotá, 1961.

\section{Cirugía oral y maxilofacial e implantología}

1. Implantología oral. Francisco Mantilla. Bogotá, 1985.

2. Tratamiento de las fracturas mandibulares. Gustavo Ulloa y Pedro Sarmiento. Cali, 1992.

3. Oseointegración. Mauricio Echeverri, Juan González, Guillermo Bernal. Bogotá, 1995.

\section{Periodoncia}

1. Periodoncia. Gustavo Barrios. Bogotá, 1989.

2. Periodoncia. Fundamentos de la Odontología. María Ferro y Mauricio Gómez. Bogotá, 2000.

3. Periodoncia para el odontólogo general. Alejandro Botero y colaboradores. Medellín, 2002.

\section{Farmacología}

1. Lecciones de materia médica y terapéutica dentales. Alberto Restrepo. Bogotá, 1895.

2. Farmacología y terapéutica odontológica. Hernán Pérez. Bogotá, 1997.

3. Bases para el manejo del dolor en Odontología. Sergio Mejía. Medellín, 1989.

\section{Estomatología}

1. Odontopatología: periodontología, estomatología. Pedro Miguel Sandino. Bogotá, 1957.

2. Biopsia y citología exfoliativa de lesiones en estomatología. Raúl Jiménez Gómez. Medellín, 1973.

3. Guías de manejo en estomatología pediátrica. Germán Hernández, Clara Acuña, Luis A. Campos y colaboradores. Bogotá, 1998.

\section{Oclusión y ATM}

1. Neurofisiología de la oclusión. Enrique Echeverri y Gisela Sencherman. Bogotá, 1989. 
2. Notas sobre la articulación temporomandibular. Raúl Jiménez Gómez. Medellín, 1989.

3. Placa neuromiorelajante. Mauricio Rubiano, Caracas. 1990.

4. Articulación temporomandibular. Hernándo Velásquez, Fanny Alvear y colaboradores. Medellín, 1992.

\section{Odontología general}

1. Estudios dentales. Guillermo Vargas Paredes. Bogotá, 1892.

2. Notas odontológicas y formulario dental. Ramón Lince Pinillos. Medellín, 1920.

3. Diagnóstico y tratamiento integral en odontología general. Ramsés Hakim y Hernando Matíz Camacho. Bogotá, 1991.

4. Urgencias odontológicas. Gustavo Malagón, Olga Malagón y colaboradores. Bogotá, 1994.

\section{Operatoria y biomateriales}

1. Operatoria dental. Paulo Emilio Herrán. Bogotá, 1910.

2. Operatoria dental moderna. Fabio Becerra y Carlos Escobar. Medellín, 1982.

3. Biomateriales, cerámica y rehabilitación oral. Humberto Guzmán y colaboradores. Bogotá, 1984.

4. Biomateriales odontológicos de uso clínico. Humberto Guzmán. Bogotá, 1990.

\section{Odontopediatría y ortopedia maxilar}

1. Asistencia dental infantil. Ramón Lince Pinillos. Medellín, 1920.

2. Ortopedia maxilar y antropología biológica. David Ordóñez. Bogotá, 1984.

3. Principios básicos de la aparatología removible. Gabriel Espinal y colaboradores. Medellín, 1995.

4. Odontología pediátrica. Darío Cárdenas. Medellín, 1996.

\section{Básicas}

1. Observaciones sobre la estructura, fisiolojía (sic), anatomía y enfermedades de los dientes. Joseph ver Valen. Bogotá, 1849.

2. Lecciones de histología y bacteriología. Sebastián Carrasquilla. Bogotá, 1908.

3. Tratado de patología y terapéutica dentales. Sebastián Carrasquilla. Bogotá, 1910. De acuerdo con Aquiles Echeverri esta obra había sido publicada en 1906, de modo que la edición de 1910 fue una versión mejorada.

4. Microbiología básica para el área de la salud y afines. Hugo Montoya Villafañe. Medellín, 2000.

\section{Prótesis y rehabilitación oral}

1. Tratado de prótesis dental. Joaquín Restrepo Tamayo. Bogotá, 1912.

2. Manual de prótesis total. Mario Villamizar y colaboradores. Bogotá, 1992.

3. Manual teórico-práctico de prostodoncia total. José V. Ricciulli. Bogotá, 1992.

4. Rehabilitación del paciente edentado. Piedad Echeverría y Miguel Roldán y colaboradores. Medellín, 1997.

5. Análisis y diseño biomecánico de la restauración parcial removible. Alfredo Quintero Ramírez. Bogotá, 2000.

6. Rehabilitación oral del paciente geriátrico. Marilia Hernández. Bogotá, 2001.

\section{Ortodoncia}

1. Ortodoncia. José Mayoral, Guillermo Mayoral y Pedro Mayoral. Barcelona, 1969.

2. Técnica ortodóncica con fuerzas ligeras. José Mayoral y Guillermo Mayoral. Barcelona, 1976.

3. Ortodoncia para el odontólogo general. Carlos Sanín y Óscar López. Medellín, 1979.

4. Ortodoncia preventiva clínica. Ricardo Aristiguieta. Bogotá, 1985.

5. Ficción y realidad en ortodoncia. Guillermo Mayoral. Bogotá, 1997.

6. Ortodoncia. Fundamentos de la odontología. Guillermo Rubio, Alejandro Zapata y colaboradores. Bogotá, 2002.

7. Cefalometría. Jaime Agudelo. Medellín, 2002.

\section{Endodoncia}

1. Dientes sin pulpa. Rafael Torres Pinzón. Bogotá, 1936.

2. Endodoncia simplificada. Gabriel Tobón y Humberto Vélez. Medellín, 1977.

3. El odontólogo general y la endodoncia. Flavio Santander. Cali, 1988.

4. Fundamentos en endodoncia. Jorge Orlando Cortés. Bogotá, 1995.

5. Endodoncia preclínica. Jorge Orlando Cortés. Bogotá, 1997.

6. Endodoncia práctica. Jorge Orlando Cortés. Bogotá, 1997.

7. Manual básico de endodoncia. Fundamentos de odontología. Diego Tobón Calle. Medellín, 2003.

\section{Ética profesional, educación y currículo}

1. Código del profesional. Álvaro Delgado Morales. Bogotá, 1928. 
2. Código del odontólogo colombiano. Álvaro Delgado Morales. Bogotá, 1936.

3. Educación, ética y legislación odontológica colombiana. Rafael Torres Pinzón. Bogotá, 1954.

4. Proceso de autoevaluación en las facultades de medicina, odontología y enfermería de Colombia. ACFO, ASCOFAME, ACOFAEN. Bogotá, 1989.

5. La enseñanza de la odontología. Carlos Payares, Alberto Arango y Juan E. Vélez. Medellín, 1990.

6. Manual de ética odontológica. Víctor H. Montes y colaboradores. Bogotá, 2001.

7. Estrategias didácticas para la enseñanza de la odontopediatría. Clara Acuña, Vilma Segura y colaboradores. Bogotá, 2002.

8. Código de conducta del cuerpo médico y odontológico. Fundación Santafé de Bogotá. Bogotá, 2002.

\section{Otros temas}

1. Manual del dentista. José Peregrino Sanmiguel. Bogotá, 1866.

2. Bibliografía dental colombiana. Alberto Patiño. Bogotá, 1910.

3. Manejo empresarial del consultorio dental. Alfredo Gánem. Bogotá, 1991.

4. Manual de asepsia y bioseguridad en odontología. Yaneth Sánchez, Leonor Quiceno y colaboradores. Medellín, 1995.

5. Odontología empírica en Colombia. Victor Hugo Montes C. Bogotá, 1995.

6. La práctica odontológica: una práctica de incertidumbre. Gonzalo Jaramillo. Medellín, 1997.

7. Manual AHO para la prevención oral. Antonio Gutiérrez. Medellín, 1999.

8. La investigación en odontología: un camino en construcción. Dora Cardona y Martha Fonseca. Bogotá, 2000. 9. Administración y auditoría odontológica. Mauricio Roldán. Bogotá, 2002.

10. Estudio epidemiológico de salud y maloclusión dental en niños de Bogotá, Colombia. Lucía Peña y Clara Gordillo. Bogotá, 2002.

\section{Historia de la odontología}

1. Reseña histórica de la odontología en Medellín. Ramón Lince Pinillos. Medellín, 1944.

2. Historia y legislación de la odontología en Colombia. Aquiles Echeverri. Buenos Aires, 1952.

3. Memorandum para la historia de la odontología en Colombia. Álvaro Delgado. Bogotá, 1964.
4. Medicina, odontología y sociedad. Carlos Payares y Raúl Jiménez. Medellín, 1980.

5. Consideraciones sociohistóricas de la odontología en Colombia y Antioquia, siglo XX. Carlos Payares y Alberto Arango. Medellín, 1991.

6. La odontología en Colombia, reseña biográfica. Benjamín Herazo Acuña. Bogotá, 1994.

7. 50 años de odontología Javeriana (1950-2000). Benjamín Herazo Acuña. (Editor). Bogotá, 2000.

8. Historia de la odontología en Córdoba. Jaime Castro Núñez. Montería, 2001.

9. La odontología en Colombia. Camilo Duque y Héctor López. Bogotá, 2002.

10. 70 años de odontología Universidad Nacional de Colombia (1932-2002). Benjamín Herazo Acuña. Bogotá, 2002.

\section{Epidemiología oral}

1. Principios de odontología sanitaria. Arturo Ocampo. Bogotá, 1958.

2. Primer Estudio Nacional de Salud Bucal. Raúl Mejía Villa, Jorge Torres, Carlos Agualimpia y Wilson Rodríguez. Bogotá, 1965 - 1971.

3. Segundo Estudio Nacional de Salud Bucal. Benjamín Herazo Acuña, Orlando Moncada. Bogotá, 19771980.

4. Aspectos epidemiológicos de la fluoruración. Jhon Flórez. Medellín, 1978.

5. Clínica del sano en odontología. Benjamín Herazo Acuña. Bogotá, 1987.

6. Fluoruros. Benjamín Herazo Acuña. Bogotá, 1988.

7. Higiene bucodental y cepillos dentales. Benjamín Herazo Acuña. Bogotá, 1990.

8. Antropología y epidemiología bucodental colombiana. Benjamín Herazo Acuña. Bogotá, 1992.

9. Cremas dentales. Benjamín Herazo Acuña. Bogotá, 1994.

10. Sellantes en odontología. Benjamín Herazo Acuña. Bogotá, 1994.

11. Diferentes temas sobre odontología y salud bucal. Víctor H. Montes. Bogotá, 1994.

12. Metrosalud. Guías de promoción, prevención y atención en salud oral. Metrosalud. Medellín, 1994.

13. El municipio sano. Benjamín Herazo Acuña. (Editor) Bogotá, 1997.

14. Odontología preventiva y social. Martha Fonseca y colaboradores. Bogotá, 1999.

15. Tercer Estudio Nacional de Salud Bucal. Ángela María Franco y colaboradores. Bogotá, 1997 - 2000. 


\begin{tabular}{lc}
\hline Área de la profesión & Cant. \\
\hline Colecciones (varios temas) & 1 \\
Odontología forense & 2 \\
Radiología & 2 \\
Cirugía oral y maxilofacial & 3 \\
e implantología & \\
Periodoncia & 3 \\
Estomatología & 3 \\
Farmacología & 3 \\
Oclusión y ATM & 4 \\
Operatoria y biomateriales & 4 \\
Odontopediatría y ortopedia maxilar & 4 \\
Odontología general & 4 \\
Básicas & 4 \\
Prótesis y rehabilitación & 6 \\
Ortodoncia & 7 \\
Endodoncia & 7 \\
Ética profesional, educación & 8 \\
y currículo & 10 \\
Otros temas & 10 \\
Historia de la odontología & 15 \\
Epidemiología & $\mathbf{1 0 0}$ \\
Total en 155 años & \\
\hline
\end{tabular}

Tabla 1. Área y cantidad de libros publicados en Colombia desde 18492004.

En cuanto al género de los autores, la tabla 2 los muestra discriminados de la siguiente forma:

- Masculino. Libros escritos por un solo autor, de género masculino.

- Masculinos. Libros escritos por varios autores, de género masculino.

- Masculinos y femeninos. Libros escritos por varios autores, de géneros femenino y masculino.

- Femeninos. Libros escritos por varios autores, de género femenino.

-Femenino. Libros escritos por un solo autor, de género femenino.

De acuerdo con esta tabla, los autores de género masculino han escrito el 79\% de los libros de odontología en Co-

\begin{tabular}{lc}
\hline Género & Cantidad \\
\hline Masculino & 61 \\
Masculinos & 18 \\
Masculinos y femeninos & 17 \\
Femeninos & 3 \\
Femenino & 1 \\
\hline
\end{tabular}

Tabla 2. Discriminación por género de autores. lombia. El género femenino ha producido el $4 \%$ y entre hombres y mujeres han escrito el 17\%.

En lo concerniente a las ciudades de publicación, la tabla 3 muestra que en Bogotá se han publicado más de la mitad de los libros odontológicos, esto es, el 67\%. Le sigue en orden la ciudad de Medellín con un 25\% y en menor proporción otras ciudades de Colombia como Manizales, Cali y Montería y ciudades del extranjero como Caracas, Buenos Aires y Barcelona. En Bogotá y Medellín se han publicado el 92\% de los libros odontológicos en Colombia.

\begin{tabular}{lc}
\hline Ciudad & Cantidad \\
\hline Bogotá & 67 \\
Medellín & 25 \\
Otras ciudades & 8 \\
\hline
\end{tabular}

Tabla 3. Discriminación por ciudades de publicación.

Al analizar la Tabla 4 se pueden observar las décadas en las que se han publicado estos ejemplares, encontrando que en los decenios de 1850, 1870 y 1880, no se publicó ningún libro y entre las décadas del cuarenta y sesenta del siglo XIX sólo se publicaron dos títulos. De 1850 a 1880 solamente se publicó un libro en Colombia sobre odontología: Manual del dentista, escrito por José Peregrino Sanmiguel y publicado en Bogotá en 1866.

En la primera mitad del siglo XX la situación no mejoró y la publicación de obras de cátedra continuó siendo más la excepción que la regla. En cincuenta años (1900-1950) sólo vieron la luz pública dieciséis libros, lo que equivale a 3.2 libros/década. Los años más significativos fueron los diez, cuando se publicaron cuatro textos. Los prolíficos cincuenta y sesenta representaron una ruptura con la primera parte de la centuria. Entre 1950 y 1960 se publicaron nueve libros, cifra nunca antes vista en el país para un período de veinte años.

En 100 años, transcurridos desde 1849 cuando se publica el primero hasta finales de 1940, sólo se publicaron quince libros; en tanto que en veinte años, transcurridos entre las décadas del cincuenta y sesenta, vieron la luz pública nueve. La década más notoria en la literatura odontológica colombiana es, hasta el momento, la de 1990, pues se publicaron 35 obras de cátedra, un volumen considerable de acuerdo con el patrón histórico. Sin embargo, en tan sólo cuatro años del presente decenio (2000 2010) se han publicado 21 libros, lo que hace pensar que ésta será la década más importante de todas. 


\begin{tabular}{cc}
\hline Década & Cantidad \\
\hline 1840 & 1 \\
1850 & 0 \\
1860 & 1 \\
1870 & 0 \\
1880 & 0 \\
1890 & 2 \\
1900 & 1 \\
1910 & 4 \\
1920 & 3 \\
1930 & 2 \\
1940 & 1 \\
1950 & 5 \\
1960 & 4 \\
1970 & 5 \\
1980 & 15 \\
1990 & 35 \\
2000 & 21 \\
\hline
\end{tabular}

Tabla 4. Discriminación por décadas.

\section{CONCLUSIONES}

- La publicación de libros odontológicos en Colombia fue -y sigue siendo- una actividad poco estimulada. - El primer impulso a la bibliografía odontológica colombiana sucedió entre los años cincuenta y sesenta. - La década del noventa ha sido la más prolífica, con 35 títulos.

- Respecto a la publicación de obras de tipo odontológico, Colombia marcha a la zaga con respecto a países de América Latina como México, Argentina y Chile.

- Si bien esta lista de libros odontológicos es la más completa que se ha publicado en Colombia, no se puede afirmar de manera categórica que este sea el número total de títulos. Con seguridad algunos textos quedaron, inadvertidamente, por fuera de este inventario; de ahí la importancia de crear una base de datos.

\section{RECOMENDACIONES}

- Crear una base de datos electrónica donde se pueda actualizar y corregir constantemente la producción bibliográfica en Colombia.

\section{AGRADECIMIENTOS}

Funcionarios de las bibliotecas de las universidades de Cartagena, del Sinú, Antioquia, CES y Javeriana.
BIBLIOGRAFÍA

1. Cardona D, Fonseca M. La investigación en odontología: un camino en construcción. Publicación de la ACFO. Bogotá, 2000.

2. Duque C, López H. La odontología en Colombia. Universidad El Bosque. Bogotá, 2002.

3. Echeverri A. Historia y legislación de la odontología en Colombia. Quetzal editores. Buenos Aires, 1952.

4. Herazo B. La odontología en Colombia, reseña biográfica. Ecoe ediciones. Bogotá, 1994.

5. Payares C, Arango A. Consideraciones sociohistóricas de la odontología en Colombia y Antioquia, Siglo XX. Promotora de ediciones y comunicaciones. Medellín, 1991.

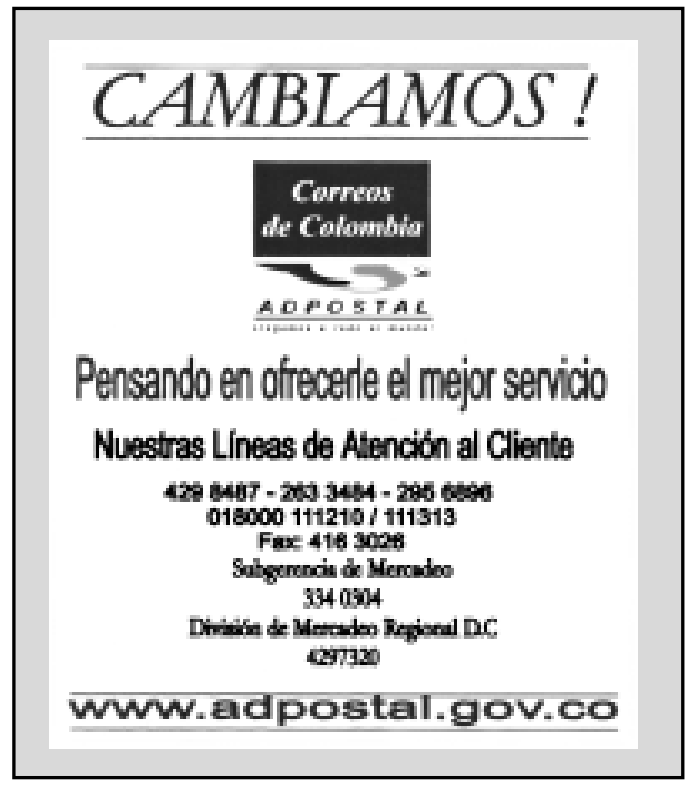

60 